\title{
High-Pass-Filtered Phase Image: Left- versus Right-Handed MR Imaging Systems
}

W e read with interest the article entitled "Detection of Intratumoral Calcification in Oligodendrogliomas by Susceptibility-Weighted MR Imaging" " and would like to comment on the appearance of calcification on the high-pass-filtered phase images.

The authors reported that the paramagnetic (authors wrote "diamagnetic") hemorrhagic component of the tumor would cause a negative phase shift and appear as dark signal on the highpass-filtered phase images, while the diamagnetic (authors wrote "paramagnetic") intratumoral calcifications would cause an opposite positive phase shift and appear as bright signal on the highpass-filtered phase images. This description is true, but only in the case of right-handed MR imaging systems, while in left-handed MR imaging systems, the complete opposite signal would be seen: Paramagnetic substances would appear bright, while diamagnetic substances would appear dark. ${ }^{2,3}$

In Figs $2 D$ and $3 D$ of the above-mentioned article, the highpass-filtered phase images are those of a left-handed MR imaging system, evident by the bright signal of the veins (paramagnetic deoxyhemoglobin). ${ }^{3}$

The article showed that high-pass-filtered phase images can depict intratumoral calcification in oligodendrogliomas better

http://dx.doi.org/10.3174/ajnr.A3571 than conventional MR images; this finding has been reported before. ${ }^{4}$ Understanding the contrast appearance of high-passfiltered phase images on left-handed versus right-handed MR imaging systems would make distinguishing diamagnetic calcification from paramagnetic hemorrhage a much easier task and prevent any possible confusion.

\section{REFERENCES}

1. Zulfiqar M, Dumrongpisutikul N, Intrapiromkul J, et al. Detection of intratumoral calcification in oligodendrogliomas by susceptibilityweighted MR imaging. AJNR Am J Neuroradiol 2012;33:858-64

2. Duyn J, Speck O. Brain Anatomy with Phase. In: Haacke EM, Reichenbach JR. Susceptibility Weighted Imaging in MRI: Basic Concepts and Clinical Applications. Hoboken, New Jersey: John Wiley \& Sons; 2011:121-36

3. Mittal S, Thomas B, Wu Z, et al. Novel approaches to imaging brain tumors. In: Haacke EM, Reichenbach JR. Susceptibility Weighted Imaging in MRI: Basic Concepts and Clinical Applications. Hoboken, New Jersey: John Wiley \& Sons; 2011:151-70

4. Wu Z, Mittal S, Kish K, et al. Identification of calcification with MRI using susceptibility-weighted imaging: a case study. J Magn Reson Imaging 2009;29:177-82

T.M. Mehemed

A. Yamamoto Department of Diagnostic Imaging and Nuclear Medicine Kyoto University Graduate School of Medicine Kyoto, Japan 